\title{
GENERALIZED WALLACE THEOREMS
}

\author{
PETER V. DANCHEV and PATRICK W. KEEF
}

\begin{abstract}
We present a number of generalizations of a classical result of Wallace regarding countable extensions of totally projective primary abelian groups.
\end{abstract}

\section{Introduction}

By the term "group" we will mean an abelian $p$-group, for some fixed prime $p$. Our terminology and notations will generally follow [10] and [13]. For instance, if $\alpha$ is an ordinal and $G$ is a group, $p^{\alpha} G$ denotes the subgroup consisting of elements of height at least $\alpha$. In particular, $p^{\omega} G$ will be the first Ulm subgroup of $G$, i.e., the set of elements of infinite height. We will also use without comment standard terminology on valuated groups and vector spaces (see, for example, [24] and [12]).

In [25], K. D. Wallace proved the following interesting result:

THEOREM 1.1. Suppose the reduced group A has a totally projective subgroup $G$ such that the quotient $A / G$ is countable. Then $A$ is totally projective.

In [3]-[8] this result was generalized to several classes of groups which properly contain the totally projectives; the goal of the present effort is to advance this investigation. In general, if $G$ is a subgroup of $A$ such that $A / G$ is countable and $\mathscr{P}$ is some property, one can ask whether $G$ satisfies $\mathscr{P}$ implies that $A$ satisfies $\mathscr{P}$, or visa versa. Similarly, one can consider the dual question, i.e., if $K$ is a countable subgroup of $G$ and $A=G / K$, does assuming that $G$ satisfies $\mathscr{P}$ imply that $A$ satisfies $\mathscr{P}$, or visa versa.

Using some terminology of [17], these two questions can be combined as follows: If $G$ and $A$ are groups, $\kappa$ is an infinite cardinal and $f: G \rightarrow A$ is a homomorphism, then $f$ is said to be $\kappa$-injective if $|K|<\kappa$, where $K$ is the kernel of $f ; f$ is said to be $\kappa$-surjective if $|C|<\kappa$, where $C=A / f(G)$ is the cokernel of $f$; and $f$ is said to be $\kappa$-bijective if it is both $\kappa$-injective and $\kappa$-surjective. This terminology, then, leads us to investigate the following type of question: If $\mathscr{P}$ is some property and $f: G \rightarrow A$ is an $\omega_{1}$-bijective

Received November 6, 2007. 
homomorphism, does $G$ satisfying $\mathscr{P}$ imply that $A$ satisfies $\mathscr{P}$, or visa versa. For example, when $\mathscr{P}$ is the property " $X$ is a separable $p^{\omega+n}$-projective group" or the property " $X$ is a $Q$-group," we show that if $f: G \rightarrow A$ is an $\omega_{1}$-bijective homomorphism, then $G$ has property $\mathscr{P}$ iff $A$ has property $\mathscr{P}$ (see Theorem 4.2 and Corollary 5.2(b), respectively).

Note that if $f: G \rightarrow A$ is an $\omega_{1}$-bijective homomorphism and $I$ is the image of $f$, then $G \rightarrow I$ is an $\omega_{1}$-bijection which is actually surjective, and $I \subseteq A$ is an $\omega_{1}$-bijection which is actually injective. This observation often allows us to split our arguments into two cases; one where $A=G / K \cong I$ is a factor group with $K$ countable and one where $G=I \subseteq A$ is a subgroup such that the cokernel $C=A / G$ is countable.

Naturally, not every property considered will allow us to generalize Wallace's Theorem in this way; it is often necessary to restrict our attention somewhat. For example, when $\mathscr{P}$ is the property " $X$ is simply presented", or the property " $\alpha \leq \omega_{1}$ and $X$ is a $C_{\alpha}$-group", and $f: G \rightarrow A$ is an $\omega_{1}$-bijective homomorphism, then if $G$ satisfies $\mathscr{P}$, it follows that $A$ satisfies $\mathscr{P}$, but the converse does not hold (Theorems 2.4 and 3.5 and Examples 2.2 and 2.3). Sometimes the natural proofs of our results use additional properties of either the kernel of $f$ as a subgroup of $G$ or the image of $f$ as a subgroup of $A$, such as requiring that it be pure, isotype or nice. We construct a number of examples to verify that these statements can fail without these additional hypotheses.

\section{Simply presented groups}

We begin with the following strengthening of Wallace's Theorem:

Proposition 2.1. Suppose $G$ and $A$ are reduced groups and $f: G \rightarrow A$ is an $\omega_{1}$-bijective homomorphism. If $G$ is totally projective, then $A$ is totally projective.

Proof. Let $I$ be the image of $f$. Since $I$ is a subgroup of $A$, it is also reduced. If we can show that $I$ is totally projective, then since $A / I$ is countable, it will follow from Wallace's Theorem that $A$ is totally projective, as well. It therefore suffices to suppose that $I=A$, so that $f$ is, in fact, surjective. Let $K$ be the kernel of $f$, so that $K$ is countable, and in fact, assume $A=G / K$. By Theorem 81.9( $\alpha)$ of [10], $G$ has a nice system, i.e, a collection of nice subgroups $\mathscr{N}$ which is closed under group unions (i.e., about $\Sigma$ ) and has the property that if $X \subseteq G$ is countable, then there is a countable $N \in \mathcal{N}$ such that $X \subseteq N$.

Let $\mathcal{N}^{\prime}=\{N / K: N \in \mathcal{N}$ and $K \subseteq N\}$. By Lemma 79.3(i) of [10] every element of $\mathcal{N}^{\prime}$ is a nice subgroup of $A=G / K$. It is easy to see that since $\mathcal{N}$ is closed under group unions, so is $\mathscr{N}^{\prime}$. Finally, if $X^{\prime} \subseteq A$ is countable, then 
$X=K \cup\left\{x \in G: x+K \in X^{\prime}\right\} \subseteq G$ is also countable; if $N \in \mathcal{N}$ is countable and $X \subseteq N$, then it follows that $N / K \in \mathscr{N}^{\prime}$ is countable and $X^{\prime} \subseteq N / K$. Therefore, $\mathscr{N}^{\prime}$ is a nice system for $A$, so that it is totally projective, as required.

It might be tempting to conjecture that the converse of this proposition holds, i.e., if $G$ and $A$ are reduced groups, $f: G \rightarrow A$ is an $\omega_{1}$-bijection and $A$ is totally projective, then $G$ is also totally projective. In fact, this fails for at least two reasons. The following shows that it can fail when $f$ is simply injective:

ExAmPLE 2.2. Suppose $A$ is a totally projective group of length $\omega_{1}$ - so $A$ is a $d s c$ group, i.e., a direct sum of countable groups - and suppose

$$
0 \rightarrow G \rightarrow A \rightarrow \mathbf{Z}_{p^{\infty}} \rightarrow 0
$$

is $p^{\omega_{1}}$-pure exact (i.e., it represents an element of $p^{\omega_{1}} \operatorname{Ext}\left(\mathbf{Z}_{p^{\infty}}, G\right)$ ). Then $A / G$ is certainly countable, but $G$ is not totally projective; in fact, it is an elementary S-group (see [26]).

Recall that if $X$ is a subgroup of a group $Y$, then $p^{\omega}(Y / X)=\cap_{i<\omega}\left(p^{i} Y+\right.$ $X) / X=\bar{X} / X$, where $\bar{X}$ is the closure of $X$ in the $p$-adic topology on $Y$. In particular, $X$ is closed iff $Y / X$ is separable. The next example shows that the converse of Proposition 2.1 can also fail when $f$ is actually surjective:

EXAmPLe 2.3. We show that there is a pure-exact (and hence isotype) sequence:

$$
0 \rightarrow K \rightarrow G \rightarrow A \rightarrow 0
$$

where $K$ is a countable direct sum of cyclics, $A$ is a dsc group of length $\omega+1$, and $G$ is a separable group which is not a direct sum of cyclics. To this end, suppose $B$ is an unbounded countable direct sum of cyclics with torsion completion $\bar{B}$ and $L$ is some group such that there is a subgroup $P \subseteq$ $L[p]$ for which $L / P$ is a direct sum of cyclics and there is an isometry $\phi$ : $\bar{B}[p] \rightarrow P$ (i.e., an isomorphism that also preserves heights computed in $\bar{B}$ and $L$ ). Since $L[p]$ is not free (as a valuated vector space), $L$ is not a direct sum of cyclics. Let $G=(B \oplus L) / X$, where $X=\{(x, \phi(x)): x \in B[p]\}$, $K=[(B \oplus\{0\})+X] / X$. Because $X$ is closed in $B \oplus L$, we can conclude $G$ is separable. Since $L$ embeds in $G$, it also follows that $G$ is not a direct sum of cyclics. It follows easily that $K \cong B$ is a pure, and hence isotype, but not nice subgroup of $G$, and that $G / K \cong L / \phi(B[p])$; we denote this last group by $A$. Note that $P / \phi(B[p])=p^{\omega}(L / \phi(B[p]))=p^{\omega} A$ is $p$-bounded and $A / p^{\omega} A=(L / \phi(B[p]) /(P / \phi(B[p]) \cong L / P$ is a direct sum of cyclics. Therefore, $A \cong G / K$ is a dsc group, and hence totally projective, as required. 
Following [10], a group is said to be simply presented if it is the direct sum of a divisible and a (reduced) totally projective group. The following gives a strengthening of Proposition 2.1 to this broader class:

THeorem 2.4. Suppose $G$ and $A$ are groups and $f: G \rightarrow A$ is an $\omega_{1-}$ bijective homomorphism. If $G$ is simply presented, then $A$ is simply presented.

We want to reduce Theorem 2.4 to Proposition 2.1. To that end, we introduce the following terminology: If $\kappa>\aleph_{0}$ is a cardinal and $G$ is reduced, then we say $G$ is a reduced $\kappa$-*-group if whenever $C$ is an infinite subgroup of $G$ with $|C|<\kappa$, then $\left|C^{\prime}\right|=|C|$, where $C^{\prime}$ is the subgroup of $G$ containing $C$ such that $C^{\prime} / C$ is the maximal divisible subgroup of $G / C$. Note that a reduced group $G$ is an $\omega_{1}$-*-group iff whenever $C$ is a countably infinite subgroup of $G$, then there is a countably infinite subgroup $C^{\prime \prime}$ of $G$ containing $C$ such that $G / C^{\prime \prime}$ is reduced; or equivalently, for all surjective homomorphisms, $g: G \rightarrow X$, with a countably infinite kernel, the maximal divisible subgroup of $X$ is also countable.

Proposition 2.5. If $G$ is a totally projective group, then $G$ is an $\omega_{1}$-*-group.

Proof. Suppose $\mathcal{N}$ is a nice system for $G$ and $C$ is a countable subgroup of $G$. Then there is a countable subgroup $N \in \mathcal{N}$ such that $C \subseteq N$. However, since $G$ is reduced and $N$ is nice, it follows that $G / N$ is reduced, as required.

An arbitrary (possibly non-reduced) group will be called a $\kappa$-*-group if it is the direct sum of a reduced $\kappa$-*-group and a divisible group. The following is the critical step in the reduction of Theorem 2.4 to Proposition 2.1.

Proposition 2.6. Suppose $G$ is an $\omega_{1}-*$-group and $A$ is a group and $f$ : $G \rightarrow A$ is an $\omega_{1}$-bijective homomorphism. Then,

(a) A is an $\omega_{1}$-*-group;

(b) If $G=G_{0} \oplus D$ and $A=A_{0} \oplus E$ where $D$ and $E$ are divisible and $G_{0}$ and $A_{0}$ are reduced, then there is an $\omega_{1}$-bijective homomorphism $f_{0}: G_{0} \rightarrow A_{0}$.

Before proving Proposition 2.6, observe how it gives the following:

Proof of Theorem 2.4. If $G=G_{0} \oplus E$ and $A=A_{0} \oplus E$, where $D$ and $E$ are divisible and $G_{0}$ and $A_{0}$ are reduced, then $G_{0}$ is totally projective, and by Proposition 2.6(b), there is an $\omega_{1}$-bijective homomorphism $f_{0}: G_{0} \rightarrow A_{0}$. By Proposition 2.1, $A_{0}$ is totally projective, so that $A$ is simply presented.

Proof of Proposition 2.6. If $K$ is the kernel of $f$ and $I$ is the image of $f$, then the obvious maps, $G \rightarrow G / K$ and $I \subseteq A$, are $\omega_{1}$-bijections with composition $f$. Therefore, if we can prove the result assuming $A=$ 
$G / K$ where $K$ is countable, and $G \subseteq A$ where $A / G$ is countable, then (a) will follow immediately and (b) will be a consequence of the fact that the composition of $\omega_{1}$-bijective homomorphisms retains that property (see, for example, Proposition 2.1(c) of [17]).

Assume first, therefore, that $K$ is a countable subgroup of $G$ and $A=$ $G / K$. Let $D=D_{1} \oplus D_{2}$, where $D_{1}$ is countable and $K \subseteq G_{0} \oplus D_{1}$. Since $A=\left(G_{0} \oplus D_{1} \oplus D_{2}\right) / K \cong\left[\left(G_{0} \oplus D_{1}\right) / K\right] \oplus D_{2}$, we may, without loss of generality, assume that $D=D_{1}$ is countable.

Note that there is an exact sequence:

$$
0 \rightarrow[D+K] / K \rightarrow A \rightarrow G /[D+K] \rightarrow 0
$$

whose left-hand group is divisible and hence a summand of $E$. If $E=([D+$ $K] / K) \oplus E_{1}$, it follows that $A_{0} \oplus E_{1} \cong G /[D+K]$. Note that $G /[D+K]=$ $\left(G_{0} \oplus D\right) /[D+K] \cong G_{0} /\left(G_{0} \cap[D+K]\right)$. Since $C=G_{0} \cap[K+D]$ is countable and $G_{0}$ is an $\omega_{1}$-*-group, there is a countable subgroup $C^{\prime}$ of $G_{0}$ containing $C$ such that $C^{\prime} / C$ is the maximal divisible subgroup of $G_{0} / C$. It follows that $G_{0} / C^{\prime} \cong\left(G_{0} / C\right) /\left(C^{\prime} / C\right)$ is isomorphic to $A_{0}$, and the obvious map $G_{0} \rightarrow G_{0} / C^{\prime} \cong A_{0}$ is an $\omega_{1}$-*-bijective homomorphism (which is actually surjective), so that (b) follows. As for (a), we need to show that this $A_{0}$ is an $\omega_{1}$-*-group. To that end, suppose $X$ is a group and $g: A_{0} \rightarrow X$ is a surjective homomorphism with countable kernel. Then the composition $G_{0} \rightarrow A_{0} \rightarrow X$ is also a surjective homomorphism with countable kernel, so, since $G_{0}$ is an $\omega_{1}$-*-group, we can conclude that the divisible part of $X$ is countable, so that $A_{0}$ is an $\omega_{1}-*$-group, as required.

Suppose next that $G \subseteq A$ with $A / G$ countable. Note $A=A_{1} \oplus D$, where $G_{0} \subseteq A_{1}$ and $A_{1} / G_{0}$ is countable. It suffices, therefore, to let $A=A_{1}$ and $G=G_{0}$, so that $G$ is reduced.

We next claim that the maximal divisible subgroup $E$ of $A$ must be countable and that $A_{0}$ is an $\omega_{1}$-*-group; before establishing the claim, note it immediately gives (a), and in addition, since the homomorphism $f_{0}: G_{0}=G \subseteq$ $A \rightarrow A / E \cong A_{0}$ is a composition of $\omega_{1}$-bijective homomorphisms, as aforementioned it is also $\omega_{1}$-bijective. This proves (b), and hence the entire result.

Turning, therefore, to the claim, suppose $C_{0}$ is an arbitrary countable subgroup of $A_{0}$, and $C_{1}$ is a countable subgroup of $A$ such that $A=G+C_{1}$. If $C=G \cap\left[C_{0}+C_{1}\right]$, then $C$ is countable, and if $C^{\prime} / C$ is the maximal divisible subgroup of $G / C$, then since $G$ is an $\omega_{1}$-*-group, $C^{\prime}$ is countable, as well. Note that $G \cap\left[C^{\prime}+C_{0}+C_{1}\right]=C^{\prime}$. It follows that

$$
\begin{aligned}
A /\left[C^{\prime}+C_{0}+C_{1}\right] & =\left[G+C_{1}\right] /\left[C^{\prime}+C_{0}+C_{1}\right] \cong G /\left(G \cap\left[C^{\prime}+C_{0}+C_{1}\right]\right) \\
& =G / C^{\prime} \cong(G / C) /\left(C^{\prime} / C\right)
\end{aligned}
$$


is reduced. This implies that $E \subseteq C^{\prime}+C_{0}+C_{1}$ is countable, giving the first part of the claim. Next, since $A_{0} /\left(A_{0} \cap\left[C^{\prime}+C_{0}+C_{1}\right]\right)$ embeds in $A /\left[C^{\prime}+C_{0}+C_{1}\right]$ and the latter is reduced, so is the former. Letting $C^{\prime \prime}=A_{0} \cap\left[C^{\prime}+C_{0}+C_{1}\right]$, we conclude that $C^{\prime \prime} \subseteq A_{0}$ is countable, $C_{0} \subseteq C^{\prime \prime}$ and $A_{0} / C^{\prime \prime}$ is reduced, showing that $A_{0}$ is an $\omega_{1}$-*-group, establishing the claim, and hence the result.

\section{3. $n$ - $\Sigma$-groups and $C_{\alpha}$-groups}

If $\alpha$ is an ordinal, then a subgroup $H$ of a group $A$ is said to be $p^{\alpha}$-high if it is maximal with respect to the property that $H \cap p^{\alpha} A=\{0\}$ (see, for example, [20] and [1]). A $p^{\omega}$-high subgroup is usually referred to simply as a high subgroup. We summarize a few standard properties of this notion in the following:

Lemma 3.1. If $\alpha$ is an ordinal and $H$ is a $p^{\alpha}$-high subgroup of $A$, then:

(a) If $\alpha<\omega$ is finite, then $H$ is a summand of $A$;

(b) If $\alpha \geq \omega$ is infinite, then $H$ is a $p^{\alpha+1}$-pure subgroup of $A$ and $A / H$ is divisible. In particular, this means that $H$ is an isotype subgroup of $A$;

(c) If $H$ is a dsc group and $H^{\prime}$ is another $p^{\alpha}$-high subgroup of $A$, then $H^{\prime}$ is also a dsc group;

(d) There is a decomposition, $A[p]=H[p] \oplus\left(p^{\alpha} A\right)[p]$;

(e) If $\alpha=\beta+\gamma$, then $p^{\beta} H$ is $p^{\gamma}$-high in $p^{\beta} A$.

Proof. (a) is Theorem 27.7 of [10]. (b) follows from $\left(2^{\circ}\right)$ and Proposition 1 of [23]. (c) is Corollary 5 of [23]. (d) and (e) are simple consequences of the maximality of $H$.

The following definition appeared in [16]: A group $A$ is a $\Sigma$-group provided that some high subgroup of $A$ is a direct sum of cyclic groups. It follows from Lemma 3.1(c) that all its high subgroups are direct sums of cyclic groups. The following generalization of this terminology was given in [18]: If $\alpha \leq \omega_{1}$, then $A$ is said to be a $C_{\alpha}$-group iff for every $\beta<\alpha$, some (and hence all) $p^{\beta}$-high subgroup of $A$ is a dsc group. If $\alpha$ is isolated, then we only need that some (and hence all) $p^{\alpha-1}$-high subgroup of $A$ is a dsc group (since if $\beta<\alpha-1$, then a $p^{\beta}$-high subgroup will be an isotype subgroup of a $p^{\alpha-1}$-high subgroup, and a classical result of Hill's states an isotype subgroup of a dsc group of countable length also has that form - see, for example, Theorem 104 of [13]). The $C_{\omega+1}$-groups are precisely the $\Sigma$-groups.

Next, we review a concept from [7]. Imitating a criterion from [2], if $1 \leq$ $n<\omega$, we shall say that $A$ is an $n-\Sigma$-group if $A\left[p^{n}\right]=\cup_{i<\omega} A_{i}$, where for all $i<\omega, A_{i} \subseteq A_{i+1}$ and $A_{i} \cap p^{i} A=\left(p^{\omega} A\right)\left[p^{n}\right]$. With this terminology, the 1 - $\Sigma$-groups are precisely the $\Sigma$-groups (this observation is generalized in our 
next result). Clearly, if $m \leq n$, then every $n$ - $\Sigma$-group is an $m$ - $\Sigma$-group. Thus each $n-\Sigma$-group is a $\Sigma$-group, while the converse implication is false (in [6], a $\Sigma$-group was constructed which is not a $2-\Sigma$-group).

Proposition 3.2. If $A$ is a group and $0<n<\omega$, then $A$ is an $n$ - $\Sigma$-group iff $A$ is a $C_{\omega+n}$-group.

Proof. Suppose $H$ is a $p^{\omega+n-1}$-high subgroup of $A$. Then $H$ is isotype in $A$, so that heights computed in $H$ and $A$ agree. By Lemma 3.1(e) and (a), $p^{\omega} H=H \cap p^{\omega} A$ is a $p^{n-1}$-high subgroup of $p^{\omega} A$, and so there is a subgroup $X \subseteq p^{\omega} A$ such that $p^{\omega} A=p^{\omega} H \oplus X$. By Lemma 3.1(d), we have $A[p]=H[p] \oplus\left(p^{\omega+n-1} A\right)[p]=H[p] \oplus X[p]$. Now, $X\left[p^{n}\right]$ is isomorphic to the direct sum of a collection of copies of $\mathbf{Z}_{p^{n}}$, and since $H$ is pure in $A$, $H\left[p^{n}\right]$ is a summand of $A\left[p^{n}\right]$. It follows that there is a decomposition:

$$
A\left[p^{n}\right]=H\left[p^{n}\right] \oplus X\left[p^{n}\right] .
$$

In fact, we claim that the above decomposition is valuated, i.e., if $z \in H\left[p^{n}\right]$ and $x \in X\left[p^{n}\right]$, then $\operatorname{ht}(z+x)=\min \{\operatorname{ht}(z)$, ht $(x)\}$ (where all heights are computed in $A$ ): Note that if $z$ has infinite height in $A$ (and $H$ ), then this follows because $p^{\omega} A=p^{\omega} H \oplus X$, and if $z$ has finite height in $A$ (and $H$ ), then this follow from $\mathrm{ht}(z)<\omega \leq \mathrm{ht}(x)$.

Suppose first that $H$ is some $p^{\omega+n-1}$-high subgroup that is a dsc group. Then $H / p^{\omega} H$ is a direct sum of cyclics, and so $H / p^{\omega} H \cong \oplus_{j<\omega} C_{j}$, where each $C_{j}$ is a direct sum of copies of $\mathbf{Z}_{p^{j+1}}$. Considering the composition:

$$
\begin{aligned}
\phi: A\left[p^{n}\right] \cong H\left[p^{n}\right] \oplus X\left[p^{n}\right] \rightarrow & H\left[p^{n}\right] \\
& \rightarrow H\left[p^{n}\right] / p^{\omega} H \subseteq H / p^{\omega} H \cong \oplus_{j<\omega} C_{j},
\end{aligned}
$$

we let $A_{i}=\phi^{-1}\left(\oplus_{j<i} C_{j}\right)$. Clearly $A_{i} \subseteq A_{i+1} \subseteq A\left[p^{n}\right]$, and since

$$
\oplus_{j<\omega} C_{j}=\cup_{i<\omega}\left(\oplus_{j<i} C_{j}\right),
$$

it follows that $A\left[p^{n}\right]=\cup_{i<\omega} A_{i}$. Next, note that all of the maps used to construct $\phi$ preserve the heights of elements whenever they are finite, and therefore, the kernel of $\phi$ is $\left(p^{\omega} A\right)\left[p^{n}\right]$. It follows that for every $i<\omega$ we have

$$
\phi\left(p^{i} A \cap A_{i}\right) \subseteq p^{i}\left(\oplus_{j<i} C_{j}\right)=\{0\} .
$$

From this we can conclude that $p^{i} A \cap A_{i}=\left(p^{\omega} A\right)\left[p^{n}\right]$, which means that $A$ is an $n$ - $\Sigma$-group.

Conversely, suppose $A$ is an $n$ - $\Sigma$-group and $H$ is any $p^{\omega+n-1}$-high subgroup of $A$. To show that $A$ is a $C_{\omega+n}$-group, we need to show that $H$ is a dsc group, or, since $p^{\omega} H$ is bounded, that the Ulm factor $H / p^{\omega} H$ is a direct sum of 
cyclics. Let the $A_{i} \subseteq A\left[p^{n}\right]$ be as in the definition of an $n$ - $\Sigma$-group. Note that $p^{\omega} H \subseteq H\left[p^{n-1}\right]$, so that $\left(H / p^{\omega} H\right)[p] \subseteq H\left[p^{n}\right] / p^{\omega} H$. In addition, for any $i<\omega, p^{\omega} H \subseteq\left(p^{\omega} A\right)\left[p^{n}\right] \subseteq A_{i}$, so we let

$$
S_{i}=\left(H / p^{\omega} H\right)[p] \cap\left(\left(A_{i} \cap H\right) / p^{\omega} H\right) .
$$

Note that if $x+p^{\omega} H \in S_{i}$, where $x \in A_{i} \cap H$, then $x \in p^{i} A$ implies that $x \in p^{i} A \cap A_{i} \cap H=\left(p^{\omega} A\right)\left[p^{n}\right] \cap H=p^{\omega} H$. Therefore, the heights (in $\left.H / p^{\omega} H\right)$ of the non-zero elements of $S_{i}$ are bounded by $i$. Since

$$
\begin{aligned}
\left(H / p^{\omega} H\right)[p] \subseteq H\left[p^{n}\right] / p^{\omega} H & =\left(A\left[p^{n}\right] \cap H\right) / p^{\omega} H \\
& =\cup_{i<\omega}\left[\left(A_{i} \cap H\right) / p^{\omega} H\right],
\end{aligned}
$$

we can conclude that

$$
\left(H / p^{\omega} H\right)[p]=\cup_{i<\omega} S_{i}
$$

However, this implies that $H / p^{\omega} H$ is a direct sum of cyclic groups, which implies that $H$ is a dsc group, as required.

Note that the above provides a non-homological proof of the fact that if one $p^{\omega+n-1}$-high subgroup of $A$ is a dsc group, then all $p^{\omega+n-1}$-high subgroups of $A$ are dsc groups.

COROLlary 3.3. If $0<n<\omega$ and $A$ is a group of length at most $\omega+n-1$, then $A$ is an $n-\Sigma$-group iff it is a dsc group.

Proof. In this case, $A$ is a $p^{\omega+n-1}$-high subgroup of itself.

A homological approach to these definitions can be given as follows: We let $A \nabla B$ denote the torsion product of the groups $A$ and $B$. This admittedly non-standard notation better reflects the multiplicative nature of the operation. If $\alpha \leq \omega_{1}$ is an ordinal, let $H_{\alpha}$ denote the generalized Prüfer group of length $\alpha$. It follows from Theorem 2 of [18] that $A$ is a $C_{\alpha}$-group iff $A \nabla H_{\alpha}$ is a dsc group (this latter characterization may be, in fact, a more natural definition of the term). The following, therefore, follows directly from Proposition 3.2:

Corollary 3.4. If $0<n<\omega$, then $A$ is an $n$ - $\Sigma$-group iff $A \nabla H_{\omega+n}$ is a dsc group.

These considerations lead to the following result:

Theorem 3.5. Suppose $\alpha \leq \omega_{1}$ is an ordinal, $G$ and $A$ are groups and $f: G \rightarrow A$ is an $\omega_{1}$-bijective homomorphism. If $G$ is a $C_{\alpha}$-group, then $A$ is also a $C_{\alpha}$-group. 
Proof. Suppose first that $\alpha<\omega_{1}$ is countable, so that $H_{\alpha}$ is countable, as well. Let $K$ and $I$ be the kernel and image of $f$, respectively. There is a long-exact sequence:

$$
0 \rightarrow K \nabla H_{\alpha} \rightarrow G \nabla H_{\alpha} \rightarrow I \nabla H_{\alpha} \rightarrow K \otimes H_{\alpha},
$$

and since the outer two groups are countable, it follows that $G \nabla H_{\alpha} \rightarrow I \nabla H_{\alpha}$ is an $\omega_{1}$-bijection. Since these both have length at most $\alpha$, they are reduced (in fact, by Lemma 64.2 of [10], $\left.p^{\alpha}\left(G \nabla H_{\alpha}\right)=\left(p^{\alpha} G\right) \nabla\left(p^{\alpha} H_{\alpha}\right)=\{0\}\right)$. In addition, since $G$ is a $C_{\alpha}$-group, $G \nabla H_{\alpha}$ is necessarily a dsc group, and hence $I \nabla H_{\alpha}$ is also a dsc group by Proposition 2.1 , so that $I$ is a $C_{\alpha}$-group. Now, if $C=A / I$, then $C$ is countable and there is a left-exact sequence:

$$
0 \rightarrow I \nabla H_{\alpha} \rightarrow A \nabla H_{\alpha} \rightarrow C \nabla H_{\alpha},
$$

Since $I \nabla H_{\alpha}$ is a dsc group, $A \nabla H_{\alpha}$ is reduced and $C \nabla H_{\alpha}$ is countable, it once again follows via Proposition 2.1 that $A \nabla H_{\alpha}$ is a dsc group, showing that $A$ is a $C_{\alpha}$-group, as required.

Finally, if $\alpha=\omega_{1}$, then $H_{\alpha}=\oplus_{\beta<\alpha} H_{\beta}$, so if $G$ is a $C_{\alpha}$-group, then $G \nabla H_{\alpha}=G \nabla\left(\oplus_{\beta<\alpha} H_{\beta}\right) \cong \oplus_{\beta<\alpha}\left(G \nabla H_{\beta}\right)$ is a dsc group, which implies that $G \nabla H_{\beta}$ is a dsc group for all $\beta<\alpha$, which implies that $A \nabla H_{\beta}$ is a dsc group for all $\beta<\alpha$, which implies that $A \nabla H_{\alpha}=A \nabla\left(\oplus_{\beta<\alpha} H_{\beta}\right) \cong \oplus_{\beta<\alpha}\left(A \nabla H_{\beta}\right)$ is a dsc group, which implies that $A$ is a $C_{\alpha}$-group.

Corollary 3.6. Suppose $n<\omega, G$ and $A$ are groups and $f: G \rightarrow A$ is an $\omega_{1}$-bijective homomorphism. If $G$ is an $n-\Sigma$-group, then $A$ is an $n-\Sigma$-group.

Notice that in Example 2.3, $A$ is a 1- $\Sigma$-group (in fact, it is a dsc group of length $\omega+1$ ), but $G$ is not a $1-\Sigma$-group (since any separable $1-\Sigma$-group is, in fact, a direct sum of cyclics). This shows that the implications in the last two results cannot be reversed, even in the case where $n=1$.

\section{4. $p^{\omega+n}$-projective groups}

The following elementary consequence of Wallace's Theorem has frequently been found useful (see, e.g., [1] and [15]). In fact, we include a separate proof since the result can be approached directly:

Corollary 4.1. Suppose A is a separable group with a subgroup $G$ such that $A / G$ is countable. Then $G$ is a direct sum of cyclic groups iff $A$ is a direct sum of cyclic groups.

Proof. Note that if $A$ is a direct sum of cyclics, it immediately follows that $G$ is, as well, so assume $G$ is a direct sum of cyclics. We may write 
$A=G+C$ for some countable subgroup $C$ of $A$. There is, therefore, a decomposition $G=G_{1} \oplus G_{2}$, where $G_{2}$ is countable and $C \cap G \subseteq G_{2}$. Thus, $C+G_{2}$ is a countable group with no elements of infinite height, being a subgroup of $A$, and hence $C+G_{2}$ is a direct sum of cyclics. We claim that $A=G_{1} \oplus\left(C+G_{2}\right)$ : Clearly $G_{1}+C+G_{2}=G+C=A$, and if $g_{1}=c+g_{2}$ (where each symbol represents an element of the corresponding subgroup), then $c=g_{1}-g_{2} \in C \cap G \subseteq G_{2}$ implies that $g_{1}=0$, proving the claim. Therefore, since $G_{1}$ and $C+G_{2}$ are direct sums of cyclics, the same will be true of $A$.

If $n<\omega$, then a group $A$ is $p^{\omega+n}$-projective iff for all groups $X$ we have $p^{\omega+n} \operatorname{Ext}(A, X)=\{0\}$ or, equivalently, $p^{n} \operatorname{Pext}(A, X)=\{0\}$. A more concrete characterization of this notion is given by Corollary 6.5 of [22], which states that $A$ is $p^{\omega+n}$-projective iff there is a subgroup $P$ of $A\left[p^{n}\right]$ such that $A / P$ is a direct sum of cyclics. One easy consequence of this is that an arbitrary subgroup of a $p^{\omega+n}$-projective is also $p^{\omega+n}$-projective. By Theorem 5 of [11], if $A$ and $A^{\prime}$ are $p^{\omega+n}$-projectives, then $A \cong A^{\prime}$ iff there is an isometry $A\left[p^{n}\right] \cong A^{\prime}\left[p^{n}\right]$. These groups have been studied extensively (e.g., [15]).

In [9], Dieudonné gave an example showing that in the last corollary, the hypothesis of countability is necessary (see, for example, [10], v. II, p. 16, Exercise 11). In fact, if $A$ is any $p^{\omega+n}$-projective group, then $A$ has a subgroup $P \subseteq A\left[p^{n}\right]$ (which must be a direct sum of cyclics) such that $A / P$ is also a direct sum of cyclics. On the other hand, there are many separable $p^{\omega+n_{-}}$ projective groups which are not direct sums of cyclics. This connection is developed in the following generalization of Corollary 4.1 (see also [3], [4], [8]):

Theorem 4.2. Suppose $n<\omega, G$ and $A$ are separable groups and $f$ : $G \rightarrow A$ is an $\omega_{1}$-bijective homomorphism. Then $G$ is $p^{\omega+n}$-projective iff $A$ is $p^{\omega+n}$-projective.

Proof. Before beginning, note that if $A$ is a separable group, then $A\left[p^{n}\right]$ will be a closed subgroup of $A$, so if $P$ is a subgroup of $A\left[p^{n}\right]$, then the $p$-adic closure, $\bar{P}$, will be contained in $A\left[p^{n}\right]$, i.e., $p^{\omega}(A / P) \subseteq A\left[p^{n}\right] / P$, which implies that $A / P$ has length at most $\omega+n$.

As usual, if $I$ is the image of $f$, then by considering the natural factorization $G \rightarrow I \rightarrow A$, we may break the argument into two cases, where $f$ is actually injective (and $\omega_{1}$-surjective), and where $f$ is actually surjective (and $\omega_{1}$-injective).

Suppose first that $f$ is injective; in fact, assume $G$ is a subgroup of $A$ and $A / G$ is countable. If $A$ is $p^{\omega+n}$-projective, it immediately follows that $G$ is, as well. Conversely, suppose $G$ is $p^{\omega+n}$-projective. Let $P$ be a subgroup of $G\left[p^{n}\right]$ 
such that $G / P$ is a direct sum of cyclics. Then $L=A / P$ must be reduced, and in fact, of length at most $\omega+n$. Since $(A / P) /(G / P) \cong A / G$ is countable, it follows from Wallace's Theorem that $L=A / P$ is a dsc group, and hence that $L / p^{\omega} L$ is a direct sum of cyclics. However, if $\bar{P}$ is the $p$-adic closure of $P$ in $A$, then $\bar{P} \subseteq A\left[p^{n}\right]$ and $A / \bar{P} \cong(A / P) /(\bar{P} / P) \cong L / p^{\omega} L$ is a direct sum of cyclics, showing that $A$ is $p^{\omega+n}$-projective.

Suppose next that $f$ is surjective; in fact, assume $K$ is a countable subgroup of $G$ with $A=G / K$. Suppose first that $G$ is $p^{\omega+n}$-projective. Let $P$ be a subgroup of $G\left[p^{n}\right]$ such that $G / P$ is a direct sum of cyclics. Let $G / P=$ $C_{1} \oplus C_{2}$, where $C_{2}$ is a countable subgroup containing $K^{\prime}=(K+P) / P$. If $P^{\prime}=(K+P) / K$, then $P^{\prime}$ is a subgroup of $A\left[p^{n}\right]$, and

$$
\begin{aligned}
A / P^{\prime} & =(G / K) /([K+P] / K) \\
& \cong G /[K+P] \\
\cong & \cong(G / P) /([K+P] / P) \\
& \cong C_{1} \oplus\left(C_{2} / K^{\prime}\right)
\end{aligned}
$$

is a dsc group. Therefore, if $\bar{P}^{\prime}$ is the $p$-adic closure of $P^{\prime}$ in $A$, then $\bar{P}^{\prime} \subseteq$ $A\left[p^{n}\right]$, and

$$
A / \bar{P}^{\prime} \cong\left(A / P^{\prime}\right) /\left(\bar{P}^{\prime} / P^{\prime}\right) \cong\left(A / P^{\prime}\right) / p^{\omega}\left(A / P^{\prime}\right)
$$

is a direct sum of cyclics, so that $A$ is $p^{\omega+n}$-projective.

Finally, suppose $A=G / K$ and $A$ is $p^{\omega+n}$-projective. Let $P$ be a subgroup of $A\left[p^{n}\right]$ such that $A / P=\oplus_{i \in I} C_{i}$ where each $C_{i}$ is cyclic. Let $P_{1} \leq G$ be the subgroup containing $K$ such that $P=P_{1} / K$; note $p^{n} P_{1} \subseteq K \leq P_{1}$ and $G / P_{1} \cong A / P=\oplus_{i \in I} C_{i}$. By a standard "back-and-forth" argument, there is a countable pure subgroup $L$ of $G$ containing $K$ and a countable subset $J \subseteq I$ such that $\left[L+P_{1}\right] / P_{1} \cong \oplus_{i \in J} C_{i}$. Note that since $L$ is a countable separable group, it is, in fact, a direct sum of cyclics. If $A^{\prime}=G / L$ and $P^{\prime}=\left[L+P_{1}\right] / L$, then

$$
\begin{aligned}
A^{\prime} / P^{\prime} & =(G / L) /\left(\left[L+P_{1}\right] / L\right) \\
& \cong G /\left[L+P_{1}\right] \\
& \cong\left(G / P_{1}\right) /\left(\left[L+P_{1}\right] / P_{1}\right) \\
& \cong \oplus_{i \in I} C_{i} / \oplus_{i \in J} C_{i} \\
& \cong \oplus_{i \in I-J} C_{i}
\end{aligned}
$$

is a direct sum of cyclics. Since $p^{n} P_{1} \subseteq L$, and hence $p^{n} P^{\prime}=0$, we can conclude that $A^{\prime}$ is $p^{\omega+n}$-projective. Now, for any group $X$, by Theorem 53.7 of [10], the pure-exact sequence

$$
0 \rightarrow L \rightarrow G \rightarrow A^{\prime} \rightarrow 0
$$


determines a corresponding right-exact sequence:

$$
\operatorname{Pext}\left(A^{\prime}, X\right) \rightarrow \operatorname{Pext}(G, X) \rightarrow \operatorname{Pext}(L, X) \rightarrow 0 .
$$

Since $L$ is a direct sum of cyclics, we have $\operatorname{Pext}(L, X)=0$, and since $A^{\prime}$ is $p^{\omega+n}$-projective we have $p^{n} \operatorname{Pext}\left(A^{\prime}, X\right)=0$. Therefore, $p^{n} \operatorname{Pext}(G, X)=0$ for all $X$, which means that $G$ is $p^{\omega+n}$-projective, as required.

\section{5. $\kappa$-Q-groups and weakly $\omega_{1}$-separable groups}

If $\kappa$ is an uncountable cardinal, then by a slight extension of the terminology of [21], we say $G$ is a $\kappa$-Q-group if for every infinite subgroup $C \subseteq G,|C|<\kappa$ implies $|\bar{C}|=|C|$, where $\bar{C}$ denotes the closure of $C$ in the $p$-adic topology on $G$ (so $\bar{C} / C=p^{\omega}(G / C)$ ). Following [21], a separable $\omega_{1}$-Q-group is said to be weakly $\omega_{1}$-separable. Finally, a separable group is a $Q$-group iff it is a $\kappa$-Q-group for all uncountable $\kappa$.

Theorem 5.1. Suppose $\kappa$ is an uncountable cardinal, $G$ and $A$ are separable groups and $f: G \rightarrow A$ is an $\omega_{1}$-bijective homomorphism. Then $G$ is a $\kappa-Q$ group iff $A$ is a $\kappa$-Q-group.

Proof. As usual, we break this into two arguments corresponding to when $f$ is assumed to be injective and surjective. Suppose first that $f$ is injective, and in fact, assume $G$ is a subgroup of $A$ with $A / G$ being countable. If $A$ is a $\kappa$-Q-group, and $C$ is an infinite subgroup of $G$ with $|C|<\kappa$, then the closure of $C$ in $G$ is contained in the closure of $C$ in $A$. Since the latter has the same cardinality as $C$, so must the former, and hence $G$ is also a $\kappa$-Q-group.

On the other hand, assume that it is $G$ that is a $\kappa$-Q-group, and that $C$ is an infinite subgroup of $A$ with $|C|<\kappa$. We can certainly expand $C$ without altering its cardinality so that $A=G+C$ and $C \cap G$ are infinite, so we assume that these two conditions hold. Note that these assumptions guarantee that $G /(C \cap G) \cong(G+C) / C=A / C$. Therefore, if $\overline{C \cap G}$ is the closure of $C \cap G$ in $G$ and $\bar{C}$ is the closure of $C$ in $A$, we have,

$$
\overline{C \cap G} /(C \cap G)=p^{\omega}(G /(C \cap G)) \cong p^{\omega}(A / C)=\bar{C} / C .
$$

Now, since $G$ is a $\kappa$-Q-group, we can conclude,

$$
|\bar{C} / C|=|\overline{C \cap G} /(C \cap G)| \leq|\overline{C \cap G}|=|C \cap G| \leq|C| .
$$

This, in turn, implies that $|\bar{C}|=|C|$, showing that $A$ is, in fact, a $\kappa$-Q-group.

Assume now that $f$ is surjective, and in fact, assume $A=G / K$, where $K$ is countable. Suppose first that $G$ is a $\kappa$-Q-group. If $C$ is an infinite subgroup of $A$ with $|C|<\kappa$, then let $C_{0}$ be the subgroup of $G$ containing $K$ defined 
by the equation $C=C_{0} / K$. If $\bar{C}$ is the closure of $C$ in $A$ and $\bar{C}_{0}$ is the closure of $C_{0}$ in $G$, then $\bar{C}_{0} / C_{0}=p^{\omega}\left(G / C_{0}\right) \cong p^{\omega}(A / C)=\bar{C} / C$ so $|\bar{C} / C|=\left|\bar{C}_{0} / C_{0}\right| \leq\left|C_{0}\right|=|C|$, so that $|\bar{C}|=|C|$, as required.

Conversely, suppose that $A$ is a $\kappa$-Q-group and $C_{0}$ is an infinite subgroup of $G$ with $\left|C_{0}\right|<\kappa$. Replacing $C_{0}$ by $C_{0}+K$ does not alter its cardinality, so we may assume $K \subseteq C_{0}$. Again, by possibly expanding $C_{0}$ without altering its cardinality, we may assume $C=C_{0} / K$ is infinite. Therefore, the argument of the last paragraph shows that $\left|\bar{C}_{0}\right|=|\bar{C}|=|C|=\left|C_{0}\right|$, as required.

Corollary 5.2. Suppose $G$ and A are separable groups and $f: G \rightarrow A$ is an $\omega_{1}$-bijective homomorphism. Then

(a) $G$ is weakly $\omega_{1}$-separable iff $A$ is weakly $\omega_{1}$-separable;

(b) $G$ is a $Q$-group iff $A$ is a $Q$-group.

Again following [21], a separable group is $\omega_{1}$-separable if every countable subset is contained in a countable summand. One might ask if the analogue to Corollary 5.2(a) holds for $\omega_{1}$-separable groups. The difficulty of this question is illustrated by two facts from [21]: Assuming Martin's Axiom (MA) and the denial of the Continuum Hypothesis $(\neg \mathrm{CH})$, if $A$ is an $\omega_{1}$-separable group of cardinality $\aleph_{1}$ and $G$ is a pure and dense subgroup of $A$ with $A / G$ countable, then $G$ is $\omega_{1}$-separable, and in fact, $A \cong G$ (Theorem 2.6). (In fact, under (MA $+\neg \mathrm{CH})$ the classes of weakly $\omega_{1}$-separable groups and $\omega_{1}$-separable groups both of cardinality $\aleph_{1}$, coincide, so a result analogous to Corollary 5.2(a) holds for this class.) Moreover, in [21] was also showed that if $G$ is a pure and closed subgroup of the separable group $A$, then $A$ is weakly $\omega_{1}$-separable iff $G$ and $A / G$ are weakly $\omega_{1}$-separable (Theorem 1.5). On the other hand, in the constructible universe ( $\mathrm{V}=\mathrm{L})$, if $A$ is an $\omega_{1}$-separable group of cardinality $\aleph_{1}$ and $A$ is not a direct sum of cyclic groups, then there is a pure subgroup $G$ of $A$ with $A / G \cong \mathbf{Z}_{p^{\infty}}$ and $G$ is not $\omega_{1}$-separable (Theorem 3.2).

\section{6. $\sigma$-summable and $\boldsymbol{n}$-Honda groups}

If $H$ is a group containing a subgroup $K$, then the height spectrum (of $K$ in $H)$ is defined to be the collection of ordinals $\left\{\mathrm{ht}_{H}(x): x \in K\right\}$. We say $K$ is height-finite if it has finite height spectrum. We begin with the following technical, but useful, lemma.

Lemma 6.1. Suppose $S$ is the height spectrum of a subgroup $K$ of a group $H$ and $F$ is a finite subgroup of $H$, and $S^{\prime}$ is the height spectrum of $F+K$. Then the set $S^{\prime} \backslash S$ is finite.

Proof. If we assume, by way of contradiction, that $S^{\prime} \backslash S$ is infinite, then there is an infinite set $\left\{f_{i}+k_{i}: i<\omega\right\}$ of elements of $F+K$ such that 
$\left\{\mathrm{ht}\left(f_{i}+k_{i}\right): i<\omega\right\}$ is an infinite set of elements of $S^{\prime} \backslash S$. Since $F$ is finite, there are distinct $i, j<\omega$ such that $f_{i}=f_{j}$ and ht $\left(f_{i}+k_{i}\right)<\operatorname{ht}\left(f_{j}+k_{j}\right)$. This implies that ht $\left(k_{i}-k_{j}\right)=\operatorname{ht}\left(\left(f_{i}+k_{i}\right)-\left(f_{j}+k_{j}\right)\right)=\min \left\{\mathrm{ht}\left(f_{i}+k_{i}\right), \operatorname{ht}\left(f_{j}+\right.\right.$ $\left.\left.k_{j}\right)\right\}=\operatorname{ht}\left(f_{i}+k_{i}\right) \in S^{\prime} \backslash S$. However, this contradicts that $k_{i}-k_{j} \in K$, so that ht $\left(k_{i}-k_{j}\right) \in S$.

The last result has the following immediate consequences:

Corollary 6.2. Suppose $H$ is a reduced group of length $\lambda, F$ and $K$ are subgroups of $H$, and $F$ is finite.

(a) If $\lambda$ is a limit ordinal and there is an ordinal $\alpha<\lambda$ such that $K \cap p^{\alpha} H=$ $\{0\}$, then there is an ordinal $\beta<\lambda$ such that $(K+F) \cap p^{\beta} H=\{0\}$.

(b) If $K$ is height-finite, then the same holds for $F+K$.

A group $A$ of length $\lambda$ is called $\sigma$-summable if $A[p]=\cup_{i<\omega} A_{i}$, where for all $i<\omega, A_{i} \subseteq A_{i+1}$ and $A_{i} \cap p^{\alpha_{i}} A=0$ for some $\alpha_{i}<\lambda$ (see [19]). Note the similarity of this property to the classical Kulikov's criterion describing when a group is a direct sums of cyclics; it follows that a separable group is $\sigma$-summable iff it is a direct sum of cyclics. It is well-known (see, for example, [19]) that all totally projective groups whose length is a limit ordinal of countable cofinality are $\sigma$-summable. More generally, if $\lambda$ is a limit ordinal of countable cofinality and $A$ is a direct sum of groups of length less than $\lambda$, then $A$ is $\sigma$-summable.

Although we can prove our next result using the original definition (cf., [3]), the following criterion, due to Hill ([14]), is slightly more convenient.

Hill's Criterion 6.3 ([14]). A group A of length $\lambda$ is $\sigma$-summable iff $A=\cup_{i<\omega} \Gamma_{i}$, where for all $i<\omega, \Gamma_{i} \subseteq \Gamma_{i+1}$ and there is an ordinal $\alpha_{i}<\lambda$ such that $\Gamma_{i} \cap p^{\alpha_{i}} A=\{0\}$.

Our next result was first established in [3]; nevertheless, we include a different, more conceptual, proof.

Proposition 6.4. Suppose $A$ is a reduced group of limit length $\lambda$ and $G$ is a $\sigma$-summable isotype subgroup of A such that $A / G$ is countable. Then $A$ is $\sigma$-summable.

Proof. Let $C$ be a countable subgroup of $A$ such that $A=C+G$. Write $C=\cup_{i<\omega} C_{i}$, where each $C_{i}$ is a finite subgroup and $C_{i} \subseteq C_{i+1}$. Referring to Hill's criterion, if $\mu$ is the length of $G$, we can write $G=\cup_{i<\omega} \Gamma_{i}$, where for every $i<\omega, \Gamma_{i} \subseteq \Gamma_{i+1}$ and there is an ordinal $\alpha_{i}<\mu$ so that $\Gamma_{i} \cap p^{\alpha_{i}} G=\{0\}$. Hence, $\Gamma_{i} \cap p^{\alpha_{i}} A=\{0\}$ and $\alpha_{i}<\lambda$ since $\mu \leq \lambda$. Therefore, if $\Gamma_{i}^{\prime}=\Gamma_{i}+C_{i}$, then $A=\cup_{i<\omega} \Gamma_{i}^{\prime}$ where for each $i<\omega, \Gamma_{i}^{\prime} \subseteq \Gamma_{i+1}^{\prime}$, and Corollary 6.2(a) implies that there is an ordinal $\beta_{i}<\lambda$ with $\Gamma_{i}^{\prime} \cap p^{\beta_{i}} A=\{0\}$. Finally, a second 
application of Hill's criterion allows us to conclude that $A$ is $\sigma$-summable, as required.

We now construct an example which shows that the hypothesis that $G$ be isotype cannot be removed. To this end, we pause for the following simple observation:

Proposition 6.5. If $A$ is group of length $\lambda$ and $\alpha<\lambda$, then $A$ is $\sigma$-summable iff $p^{\alpha} A$ is $\sigma$-summable.

Proof. Suppose $\lambda=\alpha+\gamma$. Assuming first that $A$ is $\sigma$-summmable, then using Hill's criterion, suppose $A$ is the union of $\Gamma_{i}$ 's, where for every $i<\omega$, there is an ordinal $\beta_{i}<\lambda$ such that $p^{\beta_{i}} A \cap \Gamma_{i}=0$. Then $p^{\alpha} A$ has length $\gamma$, and by setting $\Gamma_{i}^{\prime}=p^{\alpha} A \cap \Gamma_{i}$, we have $p^{\alpha} A=\cup_{i<\omega} \Gamma_{i}^{\prime}$. If $\beta_{i}^{\prime}=\beta_{i}-\alpha$ when $\beta_{i} \geq \alpha$ and $\beta_{i}^{\prime}=0$ when $\beta_{i}<\alpha$, then $\beta_{i}^{\prime}<\gamma$ and $p^{\beta_{i}^{\prime}}\left(p^{\alpha} A\right) \cap \Gamma_{i}^{\prime} \subseteq p^{\beta_{i}} A \cap \Gamma_{i}=0$, as required.

Conversely, if $p^{\alpha} A$ is $\sigma$-summable, then $\left(p^{\alpha} A\right)[p]=\cup_{i<\omega} A_{i}$, where for all $i<\omega, A_{i} \subseteq A_{i+1}$ and $A_{i} \cap p^{\alpha_{i}}\left(p^{\alpha} A\right)=0$ for some $\alpha_{i}<\gamma$. If $A[p]$ is the valuated direct sum $V \oplus\left(p^{\alpha} A\right)[p]$, and $A_{i}^{\prime}=V \oplus A_{i}$, then $A[p]=\cup_{i<\omega} A_{i}^{\prime}$, where for all $i<\omega, A_{i}^{\prime} \subseteq A_{i+1}^{\prime}$ and if $\alpha_{i}^{\prime}=\alpha+\alpha_{i}<\alpha+\gamma=\lambda$, then $A_{i}^{\prime} \cap p^{\alpha_{i}^{\prime}} A=0$.

Example 6.6. Let $\bar{B}$ be an unbounded torsion-complete group with $B$ a countable direct sum of cyclic groups. One easily constructs a group $L$ such that $p^{\omega} L=\bar{B}$ and $L / p^{\omega} L$ is countable. (For example, if $C$ is a countable group with $p^{\omega} C=B$, then we can let $L$ be the torsion subgroup of the completion of $C$ in the $\omega \cdot 2$ topology, i.e., the topology using $\left\{p^{\omega+i} C: i<\omega\right\}$ as a neighborhood base of 0 . Alternatively, this follows from Theorem 76.1 of [10].) Suppose now that $M$ is any group such that $p^{\omega} M$ is an unbounded direct sum of cyclics. If we let $A=M \oplus L$ and $G=M \oplus p^{\omega} L=M \oplus \bar{B}$, then it is easy to see that the following properties hold:

(1) Both $A$ and $G$ have length $\omega \cdot 2=\omega+\omega$.

(2) $G$ is a (non-isotype) subgroup of $A$ and $A / G \cong L / \bar{B}=L / p^{\omega} L$ is countable.

(3) $G$ is $\sigma$-summable (this follows from Proposition 6.5, since $p^{\omega} G=p^{\omega} M$ is a direct sum of cyclics and hence $\sigma$-summable).

(4) $A$ is not $\sigma$-summable (this also follows from Proposition 6.5, since $p^{\omega} A=p^{\omega} M \oplus \bar{B}$ is not a direct sum of cyclics and hence not $\sigma$ summable).

If $0<n<\omega$, then a reduced group $A$ will be called $n$-Honda if $A\left[p^{n}\right]=$ $\cup_{i<\omega} A_{i}$, where for every $i<\omega, A_{i} \subseteq A_{i+1}$ and $A_{i}$ is height-finite in $A$. 
Clearly, an $n$-Honda group is $m$-Honda for all $m \leq n$. Since an $n$-Honda group clearly has countable length, by Honda's criterion (see, for instance, [10], Theorem 84.1), being 1-Honda is equivalent to the usual notion of summability, and therefore, for any $n \geq 1$, an $n$-Honda group must be summable. There exists a summable group of length less than $\omega \cdot 2$ which is not 2-Honda (see [5] and [6]). Notice also that summable groups of countable limit length are themselves $\sigma$-summable.

Proposition 6.7. Suppose A is a reduced group such that $G$ is an isotype subgroup of $A$ and $A / G$ is countable. Then $G$ is $n$-Honda iff $A$ is $n$-Honda.

Proof. Note that if $A$ is $n$-Honda and $G$ is an arbitrary isotype subgroup of $A$, it easily follows that $G$ is $n$-Honda (if $A_{i}$ satisfies the definition for $A$, then one easily checks $G_{i}=A_{i} \cap G$ satisfies the definition for $G$ ). So assume it is $G$ that is $n$-Honda (and of course, $A / G$ is countable).

Observe that $A\left[p^{n}\right] / G\left[p^{n}\right] \cong(A / G)\left[p^{n}\right]$ is at most countable. Hence $A\left[p^{n}\right]=G\left[p^{n}\right]+C$, where $C \leq A\left[p^{n}\right]$ is countable. Let $G\left[p^{n}\right]=\cup_{i<\omega} G_{i}$, where for each $i<\omega, G_{i} \subseteq G_{i+1}$ and $G_{i}$ are height-finite in $G$, whence in $A$. In addition, let $C=\cup_{i<\omega} C_{i}$, where for each $i<\omega, C_{i} \subseteq C_{i+1}$ and $C_{i}$ is finite. Then $A\left[p^{n}\right]=\cup_{i<\omega} A_{i}$, where $A_{i}=G_{i}+C_{i}$. Certainly, $A_{i} \subseteq A_{i+1}$ and by Corollary 6.2(b) we have that all $A_{i}$ are height-finite in $A$. So, $A$ is $n$-Honda, finishing the proof.

Our final example shows that in Proposition 6.7, the requirement that $G$ be isotype in $A$ cannot be omitted.

Example 6.8. As in Example 6.6, let $L$ be a group such that $p^{\omega} L=\bar{B}$ where $\bar{B}$ is an unbounded torsion-complete group with $B$ a countable direct sum of cyclic groups, and such that $L / p^{\omega} L$ is countable. Next, let $G$ be a group so that $\bar{B} \subseteq G, p^{\omega} G=\bar{B}[p]$ and $G / \bar{B}$ is a direct sum of cyclic groups. (To construct such a $G$, let $H$ be a dsc group of length $\omega+1$ such that there is a group isomorphism $\phi: p^{\omega} H \rightarrow \bar{B}[p]$, and let $G=[H \oplus \bar{B}] /\{(x, \phi(x))$ : $x \in p^{\omega} H$, so $G$ is the sum of $H$ and $\bar{B}$ along $\phi$.) Finally, let $A$ be the result of identifying $\bar{B}$ in $L$ and $G$, that is, $A=L+G$ with $L \cap G=\bar{B}$. We therefore have the following:

(1) $A / G \cong L / \bar{B}=L / p^{\omega} L$ is countable.

(2) $G$ is summable (= 1-Honda): Indeed, since $G / \bar{B}$ is a direct sum of cyclics, we may write $G=\cup_{i<\omega} G_{i}$, where for each $i<\omega$ we have $\bar{B} \subseteq G_{i} \subseteq G_{i+1}$ and $G_{i} \cap p^{i} G \subseteq \bar{B}$. It follows that $G[p]=\cup_{i<\omega} G_{i}[p]$ with $G_{i}[p] \cap p^{i} G \subseteq \bar{B}[p]=\left(p^{\omega} G\right)[p]$. But $p^{\omega+1} G=0$ and therefore all $G_{i}[p]$ are height-finite in $G$. So, by Honda's criterion, $G$ is summable.

(3) $A$ is not summable (=1-Honda): Observe that $\bar{B}=p^{\omega} L \subseteq p^{\omega} A$ and $A / \bar{B}=(G / \bar{B}) \oplus(L / \bar{B})$ is a direct sum of cyclics, so that $p^{\omega} A=\bar{B}$. 
Since $p^{\omega} A=\bar{B}$ is not summable, it follows that $A$ is not summable, as claimed. Since $G \cap p^{\omega} A=\bar{B} \neq \bar{B}[p]=p^{\omega} G, G$ is not isotype in $A$.

ACKNOWLEDGMENTS. The authors would like to thank the referee for his or her valuable comments and suggestions, as well as the Editor, Professor Arne Meurman, for his efforts and patience in processing our work.

\section{REFERENCES}

1. Benabdallah, K., Irwin, J., and Rafiq, M., N-high subgroups of abelian p-groups, Arch. Math. (Basel) 25 (1974), no. 1, 29-34.

2. Danchev, P., Commutative group algebras of abelian $\Sigma$-groups, Math. J. Okayama Univ. 40 (1998), no. 2, 77-90.

3. Danchev, P., Countable extensions of torsion abelian groups, Arch. Math. (Brno) 41 (2005), no. 3, 265-273.

4. Danchev, P., A note on the countable extensions of separable $p^{\omega+n}$-projective abelian $p$ groups, Arch. Math. (Brno) 42 (2006), no. 3, 251-254.

5. Danchev, P., Abelian groups with a nice basis, Compt. Rend. Acad. Bulg. Sci. 60 (2007), no. 3, 219-224.

6. Danchev, P., Nice bases for primary abelian groups, Ann. Univ. Ferrara, Sec. Math. 53 (2007), no. 1, 39-50.

7. Danchev, P., Primary abelian $n$ - $\Sigma$-groups, Liet. Mat. Rink. 47 (2007), no. 2, 155-162.

8. Danchev, P., Notes on countable extensions of $p^{\omega+n}$-projectives, Arch. Math. (Brno) 44 (2008), no. 1, 37-40.

9. Dieudonné, J., Sur les p-groupes abeliens infinis, Portugal. Math. 11 (1952), no. 1, 1-5.

10. Fuchs, L., Infinite Abelian Groups, volumes I \& II, Academic Press, New York, 1970 and 1973.

11. Fuchs, L., On $p^{\omega+n}$-projective abelian p-groups, Publ. Math. Debrecen 23 (1976), 309-313.

12. Fuchs, L., Vector spaces with valuations, J. Algebra 35 (1978), 23-38.

13. Griffith, P., Infinite Abelian Group Theory, The University of Chicago Press, Chicago and London, 1970.

14. Hill, P., A note on $\sigma$-summable groups, Proc. Amer. Math. Soc. 126 (1998), no. 11, 3133-3135.

15. Irwin, J., Snabb, T., and Cutler, D., On $p^{\omega+n}$-projective p-groups, Comment. Math. Univ. St. Pauli 35 (1986), no. 1, 49-52.

16. Irwin, J., and Walker, E., On N-high subgroups of abelian groups, Pacific J. Math. 11 (1961), no. 4, 1363-1374.

17. Keef, P., Partially decomposable primary abelian groups and the generalized core class property, in "Models, Modules and Abelian Groups", de Gruyter, Berlin 2008, pp. 293303.

18. Keef, P., On iterated torsion products of abelian p-groups, Rocky Mountain J. Math. 21 (1991), no. 3, 1035-1055.

19. Linton, R., and Megibben, C., Extensions of totally projective groups, Proc. Amer. Math. Soc. 64 (1977), no. 1, 35-38.

20. Megibben, C., On high subgroups, Pacific J. Math. 14 (1964), no. 4, 1353-1358.

21. Megibben, C., $\omega_{1}$-separable p-groups, in Proc. $3^{\text {rd }}$ Conf., Oberwolfach (FRG 1985), Abelian Group Theory (New York), 1987, pp. 117-136.

22. Nunke, R., Purity and subfunctors of the identity, Topics in Abelian Groups, Scott, Foresman and Co., 1962, pp. 121-171.

23. Nunke, R., On the structure of Tor II, Pacific J. Math. 22 (1967), 453-464. 
24. Richman, F., and Walker, E., Valuated groups, J. Algebra 56 (1979), no. 1, 145-167.

25. Wallace, K., On mixed groups of torsion-free rank one with totally projective primary components, J. Algebra 17 (1971), no. 4, 482-488.

26. Warfield, R., A classification theorem for abelian p-groups, Trans. Amer. Math. Soc. 210 (1975), no. 1, 149-168.

DEPARTMENT OF MATHEMATICS

PLOVDIV UNIVERSITY

"P. HILENDARSKI"

PLOVDIV 4000

BULGARIA

E-mail: pvdanchev@yahoo.com
DEPARTMENT OF MATHEMATICS

WHITMAN COLLEGE

WALLA WALLA, WA, 99362

USA

E-mail: keef@whitman.edu 